\title{
Effects of spironolactone on extrasystoles and heart rate variability in haemodialysis patients: a randomised crossover trial
}

\author{
Michael Eklund ${ }^{a}$, Olof Hellberg ${ }^{a}$, Hans Furuland ${ }^{b}$, Yang Cao ${ }^{c, d}$ and Erik Nilsson ${ }^{e, f}$ \\ aDepartment of Internal Medicine, School of Medical Sciences, Örebro University, Örebro, Sweden; ${ }^{b}$ Department of Medical Sciences, \\ Uppsala University Hospital, Uppsala, Sweden; 'CClinical Epidemiology and Biostatistics, School of Medical Sciences, Örebro University, \\ Örebro, Sweden; 'Unit of Integrative Epidemiology, Institute of Environmental Medicine, Karolinska Institutet, Stockholm, Sweden; \\ eDepartment of Medical Epidemiology and Biostatistics, Karolinska Institutet, Stockholm, Sweden; fSchool of Medical Sciences, Örebro \\ University, Örebro, Sweden
}

\begin{abstract}
Background: Spironolactone treatment reduces mortality in haemodialysis (HD) patients. The objective of this study was to evaluate if spironolactone affects cardiac electric activity in this population.

Methods: Participants were randomised to start with spironolactone 50 mg daily or observation (12 weeks) with subsequent washout ( 6 weeks) and crossover to the other intervention (12 weeks). Long-term electrocardiograms were recorded and assessed with blinding to treatment. The primary outcome was premature ventricular complexes (PVC), and secondary outcomes were atrial premature contractions (APC) and heart rate variability (HRV).

Results: Thirty participants were recruited, and data for 16 participants were included in the analysis. Treatment was associated with an increase in PVCs by 9.7 [95\% confidence interval (CI): 1.5 to 18] $\mathrm{h}^{-1}$. HRV time-domain variables increased during treatment, the standard deviation of all beat-to-beat intervals by 18 (95\% Cl: 3.3 to 32) milliseconds ( $\mathrm{ms}$ ) and the standard deviation of the averages of beat-to-beat intervals in all 5-min segments of the entire recording by $16(95 \% \mathrm{Cl}: 1.5$ to 30$) \mathrm{ms}$. There were no significant differences in other variables.

Conclusion: Spironolactone treatment increases PVCs in HD, indicating a possible proarrhythmic effect. However, improved cardiac autonomic function, as indicated by an increased HRV, may contribute to the survival benefit from spironolactone treatment in HD patients.
\end{abstract}

\section{ARTICLE HISTORY}

Received 11 September 2020

Revised 16 October 2020

Accepted 10 November 2020

Published 25 January 2021

\section{KEYWORDS}

Haemodialysis

spironolactone; clinical

trial; long-term ECG;

arrhythmias; heart rate

variability

\section{Introduction}

Cardiovascular disease is a leading cause of morbidity and mortality in end-stage renal disease (ESRD) (1). In two recent trials, treatment with the mineralocorticoid receptor antagonist (MRA) spironolactone improved survival and reduced the risk of cardiovascular events in haemodialysis (HD) patients $(2,3)$. However, the mechanisms by which MRAs may improve survival in $\mathrm{HD}$ patients are not clear.

Multiple studies suggest a link between the mineralocorticoid aldosterone and cardiac electric activity (4-6). Animal studies show that overexpression of the mineralocorticoid receptor in cardiac myocytes increases the occurrence of ventricular arrhythmias (6). It also reduces heart rate variability (HRV) (6), which is a marker for autonomic function (7). In line with this, MRAs decrease atrial arrhythmias in rodents (5). A reduction in $\mathrm{HRV}$, as well as the presence of premature ventricular complexes (PVCs) and atrial premature contractions (APCs), has been associated with an increased risk of death due to cardiovascular events (8-11). These electrocardiogram (ECG) markers are also associated with the risk of arrhythmias (10,12-14).

This study aimed to investigate the effects of MRA on cardiac electrical activity in HD patients. We hypothesised that spironolactone treatment would be associated with a change in the number of PVCs on long-term ECG (LTECG).

\section{Materials and methods}

This was an open-label, randomised crossover trial controlled using untreated observation with blinded assessment of outcomes. The primary outcome was the frequency of PVCs, and secondary outcomes were the frequency of APCs and change in HRV indices.

Patients were recruited in Sweden, from the dialysis units at Örebro University Hospital and Uppsala University Hospital,

CONTACT Michael Eklund michael.eklund@regionorebrolan.se

(1) Supplemental data for this article can be accessed here.

(c) 2021 The Author(s). Published by Upsala Medical Society.

This is an Open Access article distributed under the terms of the Creative Commons Attribution License (http://creativecommons.org/licenses/by/4.0/), which permits unrestricted use, distribution, and reproduction in any medium, provided the original work is properly cited. 
from February 2013 until December 2014. Inclusion criteria were age 18 years or older, three or more weekly HD-sessions, compliance during the previous three months and adequate cognitive function. Exclusion criteria were cardiac pacemaker, persistent or permanent atrial fibrillation, plasma potassium $>6.5 \mathrm{mmol} / \mathrm{L}$ at any time during the two months preceding inclusion, life-expectancy of less than 12 months, and pregnancy or breastfeeding.

Power calculation was based on PVCs and yielded a minimum of 14 subjects completing the crossover study. The analysis was based on results from trials in congestive heart failure (15-17) and used a two-sided significance level of $5 \%$ and $80 \%$ power. The sample size was set to 30 as a dropout rate of $50 \%$ was expected, mainly due to renal transplantation, mortality and side effects of spironolactone.

Randomisation and allocation were conducted by a person not otherwise involved in the study. Sequence generation in blocks of six with a ratio of 1:1 was performed using Clinstat (Martin Bland, United Kingdom). Sequentially numbered, sealed and opaque envelopes were used for allocation.

Subjects were assigned to a sequence of 12 weeks $50 \mathrm{mg}$ oral spironolactone daily, followed by 6 weeks washout phase and finishing with 12 weeks of no treatment, or the reverse order. Spironolactone and its active metabolites have half-lives of 1.4-16.5 $\mathrm{h}$ in healthy individuals, but the effects of chronic kidney disease and HD on the elimination of spironolactone are unknown (18). The lack of knowledge on the elimination of spironolactone in the study population motivated the use of a long washout phase.

The four study visits took place on midweek dialysis sessions and were conducted before and after both of the 12week intervention periods. Blood draw and measurements were performed before dialysis treatment. Blood pressure was measured using an automated oscillometric blood pressure monitor (Omron M6 AC, Omron Healthcare, Japan). The accredited local hospital laboratories analysed routine biochemical parameters. LTECG recordings were started at the onset of the dialysis session and continued for a minimum of 24 h, using a Medilog AR12 Plus ECG recorder (Schiller AG, Switzerland). The Swedish Renal Registry was accessed to obtain information on the primary renal disease of the participants.

A clinical laboratory scientist and a physician, blinded to treatment allocation, curated the LTECG recordings, using Medilog Darwin 2 Enterprise v 2.1.0 software (Schiller AG, Switzerland). HRV analysis was conducted using R-statistics v 3.4.1 (R Development Core Team, Austria) and the package RHRV v 3.0.7 (19). HRV variables were defined in accordance with Malik et al. (7) as follows: Time-domain variables included the standard deviation of all beat-to-beat intervals (SDNN), the standard deviation of the averages of beat-tobeat intervals in all 5-min segments of the entire recording (SDANN) and the square root of the mean of the sum of the squares of differences between adjacent beat-to-beat intervals (RMSSD). Frequency-domain variables were based on power in different frequency ranges, 0.15-0.4 Hz - high frequency (HF), 0.04-0.15 Hz - low frequency (LF), 0.0030.04 - very low frequency (VLF) and $\leq 0.003 \mathrm{~Hz}$ - ultra-lowfrequency (ULF).

After treatment, remaining spironolactone tablets were collected. Compliance was calculated as the ratio of tablets consumed, based on the amount returned, to expected consumption.

Baseline data were expressed as median and interquartile range, or as percentage. A generalised linear mixed model with random patient effects was used to assess the treatment effect in all statistical analyses, as described by Senn (20). Period effects and baseline values were considered fixed effects. Log transformation to normality was used when mandated by the assessment of model residuals. All analyses were conducted using R-statistics v 3.4.1 (R Development Core Team, Austria). Per-protocol analysis with listwise deletion of incomplete cases was pre-specified in the study analysis plan, motivated by the study aim of elucidating MRAs mechanisms of action rather than to assess clinical efficacy.

The study received ethical approval by The Regional Ethical Review Board in Uppsala, Sweden (reference number 2011/316). The board decision covers both study centres of this trial. All participants gave written informed consent before enrolment. The Declarations of Helsinki was adhered to, and Good Clinical Practice was applied. The trial was approved by the Swedish Medical Products Agency (EudraCTnr 2011-002773-39) and was registered on 28 September 2011 at http://www.clinicaltrialsregister.eu/ (EudraCTnr 2011-00277339). Monitoring was performed to ensure adherence to the study protocol, complete documentation and complete registration of adverse events.

\section{Results}

\section{Baseline characteristics}

A total of 30 participants were recruited, of which 18 (60\%) were enrolled in Örebro and 12 (40\%) in Uppsala. Of these, 18 persons (60\%) completed follow-up (Figure 1). There was a larger proportion of participants completing follow-up in Örebro [13, (72\%)] than in Uppsala [5, (42\%)]. Two participants had corrupt LTECG data files from single recordings and were excluded, resulting in a total of 16 participants (53\%) included in analysis. Of the analysed population, the median age was 66 (61.5-67) years, and 12 (75\%) were male. Only two (13\%) of the included participants were current tobacco users, and three (19\%) participants were previous tobacco users. Eight participants $(50 \%)$ were treated with angiotensin-converting enzyme inhibitors or angiotensin receptor blockers (Table 1). Further, eight persons (50\%) were treated with a betaadrenoceptor antagonist. No other class of anti-arrhythmic medication was used. The potassium binder polystyrene sulphonate was used at inclusion in two cases (12\%), and one participant $(6.2 \%)$ was treated with potassium chloride supplementation. Concomitant medications have been listed in Supplementary Table 1. 


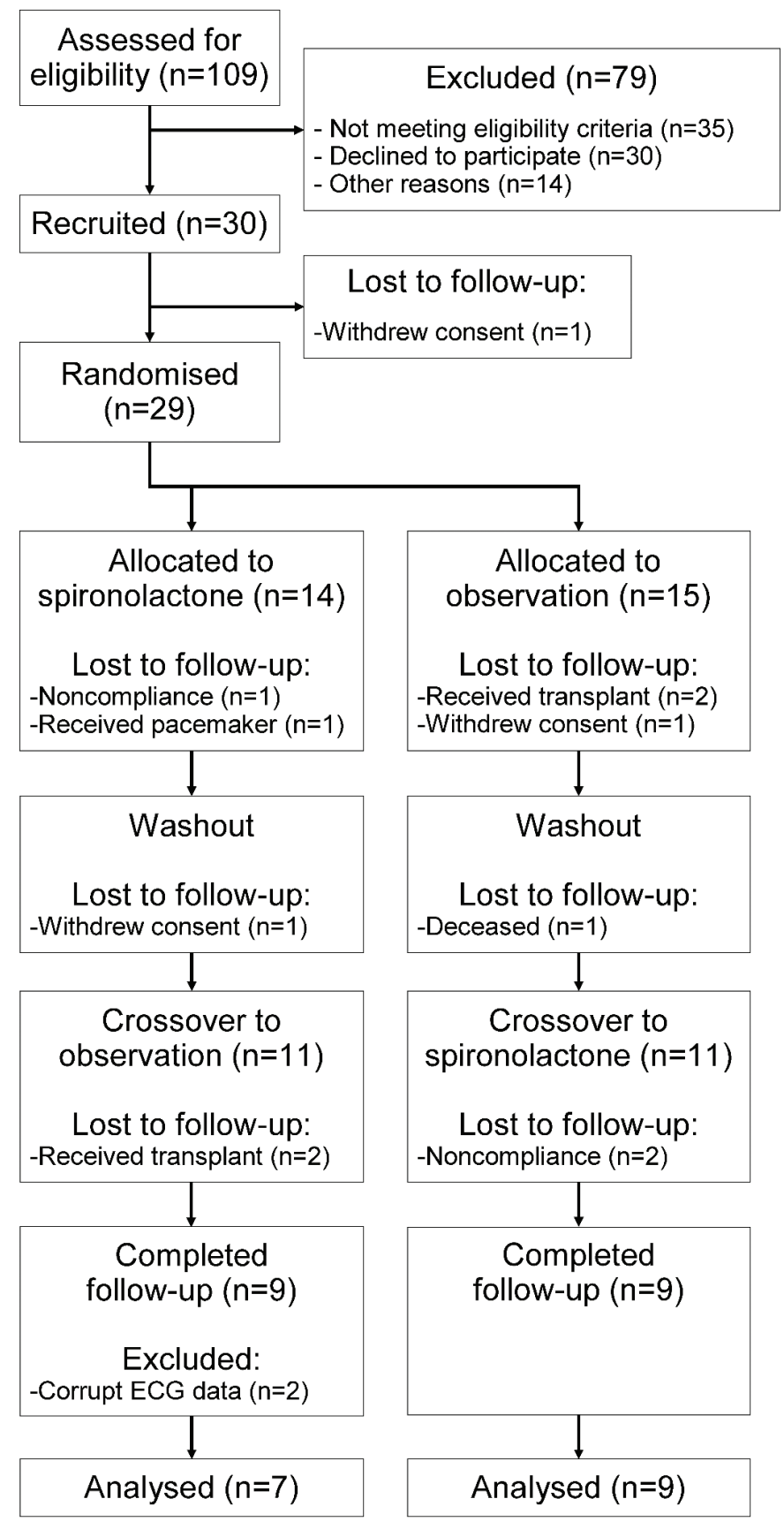

Figure 1. Flow chart of the study procedure. Study design, recruitment of participants and follow-up for long-term electrocardiogram data.

\section{Outcomes}

The 16 participants included in the analysis had a mean compliance of $97 \%$. Spironolactone treatment was associated with an increase in PVCs compared to observation [mean treatment effect $9.7,95 \%$ confidence interval (Cl): 1.5 to $18 \mathrm{~h}^{-1}$. There was no significant difference in APCs during treatment compared to observation (ratio $2.5,95 \% \mathrm{Cl}: 0.58$ to 11 ). All HRV time-domain variables (SDNN, SDANN, RMSSD) increased during treatment, although statistical significance was reached only for SDNN, which increased by 18 (95\% Cl: 3.3 to 32) milliseconds (ms), and SDANN, which increased by $16(95 \% \mathrm{Cl}$ : 1.5 to 30 ) ms (Table 2). The effects of spironolactone on these variables on the individual level are presented in Figure 2. HRV frequency-domain variables (HF, LF, VLF, ULF) did not change significantly during treatment compared to observation (Table 3). Note that in Tables 2 and 3, the LTECG variables measured at the end of the spironolactone treatment period were compared to the measurement after the observation period, and since the estimate was adjusted for both baselines, it corresponds to the change in each variable that is due to spironolactone treatment.

During the study period, the following changes in antiarrhythmic treatment were made: Three participants initiated beta-blocker treatment (bisoprolol in all cases), three discontinued treatment (two cases of atenolol, and one case of metoprolol) and three had a reduction in dose (bisoprolol in one case, and metoprolol in two cases). All changes, except a dose reduction of metoprolol for one participant, occurred during the non-intervention period of follow-up.

There was no apparent effect of spironolactone on plasma potassium concentrations, with an estimated mean difference in plasma potassium between the end of treatment and the end of observation of $-0.046 \mathrm{mmol} / \mathrm{L}(95 \% \mathrm{Cl}:-0.57$ to 0.47 ). Potassium binder polystyrene sulphonate was initiated for one participant during spironolactone treatment, and no other changes in potassium binder treatment occurred during followup. Serum bicarbonate concentration decreased by $-2.0(95 \%$ $\mathrm{Cl}:-3.5$ to -0.52$) \mathrm{mmol} / \mathrm{L}$ during treatment. Effects on electrolytes have been presented in Supplementary Table 2 .

There were no changes in systolic blood pressure $(13 \mathrm{mmHg}$, $95 \% \mathrm{Cl}:-1.8$ to 28$)$ or diastolic blood pressure $(5.6 \mathrm{mmHg}, 95 \%$ $\mathrm{Cl}-1.1$ to 12 ) during treatment compared to observation (Supplementary Table 2).

During the study, 62 adverse events were reported for the study population that underwent at least one study visit $(n=29)$. One serious adverse event was reported, with a participant passing away during the wash-out period of follow-up. The case review did not indicate any association between death and exposure to the study drug. One case of muscular cramps was considered associated with spironolactone treatment. All other events were judged as not being associated with the intervention. The most common adverse events were infections (12 occurrences), chronic obstructive pulmonary disease exacerbations (10 occurrences) and hypotension (four occurrences, of which two were during spironolactone treatment).

\section{Discussion}

The major novel findings of this study were that spironolactone treatment is associated with an increased occurrence of PVCs and that it increases HRV time-domain indices in persons undergoing HD. Although an increase in PVCs during treatment was unexpected, effects on ECG variables are in line with mineralocorticoid receptor action on cardiac electrical conductivity (4-6). Spironolactone has been shown to act as a partial mineralocorticoid receptor agonist during certain intracellular conditions in vitro $(21,22)$, and we speculate that 
Table 1. Baseline characteristics.

\begin{tabular}{|c|c|c|c|c|c|}
\hline Variable & & & & & \\
\hline Myocardial infarction & 4 & 25 & & & \\
\hline Congestive heart failure & 4 & 25 & Heart rate, $\mathrm{min}^{-1}$ & 76 & 11 \\
\hline Arrhythmia & 2 & 12 & Systolic blood pressure, $\mathrm{mmHg}$ & 140 & 30 \\
\hline Peripheral vascular disease & 3 & 19 & Diastolic blood pressure, $\mathrm{mmHg}$ & 72 & 18 \\
\hline Diabetes mellitus & 7 & 44 & Premature ventricular complex, $\mathrm{h}^{-1}$ & 2.7 & 7.2 \\
\hline Diabetes mellitus, type 1 & 1 & 6.2 & Atrial premature contraction, $\mathrm{h}^{-1}$ & 4 & 21 \\
\hline Diabetes mellitus, type 2 & 6 & 38 & Heart rate variability (HRV) Time-domain & & \\
\hline Other comorbidities & & & $\begin{array}{l}\text { Standard deviation of all beat-to-beat } \\
\text { intervals (SDNN), milliseconds (ms) }\end{array}$ & 110 & 43 \\
\hline Dyslipidaemia & 11 & 69 & $\begin{array}{l}\text { Standard deviation of the averages of } \\
\text { beat-to-beat intervals in all } 5 \mathrm{~min} \text { segments } \\
\text { of the entire recording (SDANN), } \mathrm{ms}\end{array}$ & 92 & 42 \\
\hline Obstructive sleep apnoea syndrome & & & HRV Frequency-domain* & & \\
\hline Biochemical data & Median & IQR & High frequency (HF), ms ${ }^{2}$ & 28 & 100 \\
\hline Potassium, $\mathrm{mmol} / \mathrm{L}$ & 4.8 & 0.76 & Low frequency (LF), $\mathrm{ms}^{2}$ & 100 & 120 \\
\hline Sodium, $\mathrm{mmol} / \mathrm{L}$ & 140 & 2.2 & Very low frequency (VLF), $\mathrm{ms}^{2}$ & 220 & 250 \\
\hline Magnesium, $\mathrm{mmol} / \mathrm{L}$ & 0.9 & 0.13 & Ultra-low-frequency (ULF), $\mathrm{ms}^{2}$ & 50 & 26 \\
\hline Calcium, mmol/L & 2.3 & 0.18 & & & \\
\hline Bicarbonate, $\mathrm{mmol} / \mathrm{L}$ & 23 & 2.8 & & & \\
\hline Phosphate, $\mathrm{mmol} / \mathrm{L}$ & 1.6 & 0.63 & & & \\
\hline Albumin, $\mathrm{g} / \mathrm{L}$ & 38 & 3 & & & \\
\hline
\end{tabular}

the relatively high dose of spironolactone used in this study may have contributed to an increased occurrence of PVCs, thus having a proarrhythmic effect. HRV reflects autonomic function (7), and the observed increase in HRV may be due to inhibition of aldosterone action in the central nervous system, leading to a reduction in cardiac sympathetic stimulation, as has been shown in animal studies (23). In individuals with congestive heart failure, sympathoexcitation has been associated with a reduction in HRV (24) and increased risk of death (25). Increased HRV, as demonstrated here, could, therefore, be a mechanism underlying the beneficial effect of MRA in HD patients $(2,3)$.

We also found an association between spironolactone treatment and a decrease in plasma bicarbonate concentration, which may be due to aldosterone action on $\mathrm{Na}^{+} / \mathrm{H}^{+}$exchange in the intestine (26) or remaining nephrons, with secondary effects on bicarbonate reabsorption.

Anti-arrhythmic effects of spironolactone have been suggested as an important mechanism of action leading to improved survival in congestive heart failure and ischemic heart disease, reducing the occurrence of PVCs $(16,27,28)$ and
APCs (16). To our knowledge, no prior studies have investigated the effect of spironolactone on these markers in ESRD. In contrast to studies on heart disease patients $(16,27,28)$, we found that MRA treatment increased the frequency of PVCs and did not demonstrate an effect on APCs. Differences in serum potassium concentrations (29), cardiovascular pathology and concomitant medications between these populations are possible explanations for these discrepancies. For example, in this study, only $25 \%$ of the participants had congestive heart failure, and $25 \%$ had a history of myocardial infarction, compared to $50-52 \%$ with previous myocardial ischemia in the congestive heart failure population (16). Furthermore, previous studies in chronic heart disease patients reported the use of angiotensinconverting enzyme inhibitors in more than $90 \%$ of cases $(16,27)$, compared to $50 \%$ using angiotensin-converting enzyme inhibitors or angiotensin receptor blockers in our study. These differences could indicate that a beneficial effect of spironolactone on APCs and PVCs is dependent on pathological processes that are less prevalent in HD patients than in congestive heart failure and ischemic heart disease, or that the effect requires a more extensive renin-angiotensin-aldosterone 


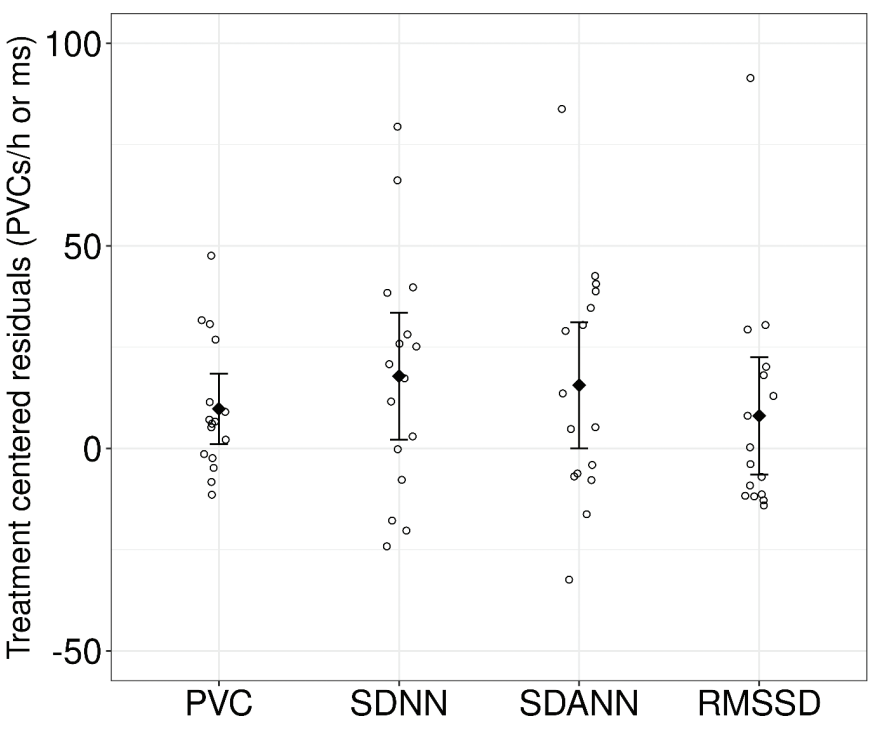

Figure 2. Effect of spironolactone on premature ventricular complexes and heart rate variability time-domain. Treatment centred residuals were calculated from the generalised linear mixed model with random effects residuals, which was added to treatment estimates, representing both treatment effect and random influences not eliminated by study design or adjustment. Diamonds signify means and horizontal bars $95 \%$ confidence intervals. Individual values are plotted as circles. Abbreviations: ms, milliseconds; PVC, premature ventricular complexes; RMSSD, the square root of the mean of the sum of the squares of differences between adjacent beat-to-beat intervals; SDANN, the standard deviation of the averages of beat-to-beat intervals in all 5-min segments of the entire recording; SDNN, the standard deviation of all beat-to-beat intervals.

Table 2. Effect of spironolactone on premature ventricular complexes and heart rate variability time-domain variables.

\begin{tabular}{lccc}
\hline Variable & Estimate & $\begin{array}{c}95 \% \text { confidence } \\
\text { interval }\end{array}$ & $p$ \\
\hline $\begin{array}{l}\text { Premature ventricular } \\
\text { complex, } \mathrm{h}^{-1}\end{array}$ & 9.7 & 1.5 to 18 & 0.024 \\
$\begin{array}{l}\text { Standard deviation of all } \\
\text { beat-to-beat intervals (SDNN), } \\
\text { milliseconds (ms) }\end{array}$ & 18 & 3.3 to 32 & 0.02 \\
$\begin{array}{l}\text { Standard deviation of the } \\
\text { averages of beat-to-beat } \\
\text { intervals in all 5-min segments of } \\
\text { the entire recording (SDANN), } \\
\text { ms }\end{array}$ & 16 & 1.5 to 30 & 0.033 \\
$\begin{array}{l}\text { Square root of the mean of the } \\
\text { sum of the squares of differences } \\
\text { between adjacent beat-to-beat } \\
\text { intervals (RMSSD), ms }\end{array}$ & 8 & -5.7 to 22 & 0.23 \\
\hline
\end{tabular}

Estimates of the treatment effect of spironolactone, corresponding to absolute change in the respective variable. Estimates, confidence intervals and $p$-values were calculated from a generalised linear mixed model with random effects, using fixed effects for treatment, intervention order and baseline values as well as random effects for patients.

system inhibition, achieved through concomitant angiotensinconverting enzyme inhibitors treatment.

A few trials have investigated the effect of spironolactone on HRV in ESRD. In a study by Flevari et al. (30), 14 HD patients without congestive heart failure were treated with spironolactone $25 \mathrm{mg}$ three times weekly for 4 months. In line
Table 3. Effect of spironolactone on heart rate variability frequency-domain variables.

\begin{tabular}{lccc}
\hline Variable* & Estimate & $\begin{array}{c}\text { 95\% confidence } \\
\text { interval (Cl) }\end{array}$ & $p$ \\
\hline High frequency (HF) & 1.8 & 0.81 to 4.1 & 0.13 \\
Low frequency (LF) & 1.3 & 0.77 to 2.3 & 0.29 \\
Very low frequency (VLF) & 1.3 & 0.94 to 1.7 & 0.11 \\
Ultra-low frequency (ULF) & 1.2 & 0.83 to 1.6 & 0.35 \\
\hline
\end{tabular}

Estimates of the treatment effect of spironolactone, corresponding to the ratio between treatment and observation. Values were log-transformed, and estimates, confidence intervals and $p$-values were calculated with a generalised linear mixed model with random effects. Treatment, intervention order and baseline values were used as covariates, and patient effects were treated as random. Estimates and confidence intervals were antilogged to get ratios.

*Power in different ranges, HF $0.15-0.4 \mathrm{~Hz}, \mathrm{LF} 0.04-0.15 \mathrm{~Hz}$, VLF $0.003-0.04 \mathrm{~Hz}$, ULF $\leq 0.003 \mathrm{~Hz}$.

with our results, the authors found that HRV increased in the time-domain variable RMSSD during treatment but also that HRV frequency-domain indices (ULF and VLF) decreased. Effects on SDNN, the most commonly used marker for cardiac autonomic function, were not reported. Lin et al. (2) conducted a trial with 253 ESRD patients, who received spironolactone $25 \mathrm{mg}$ daily, or placebo, for two years. Contrary to our results, spironolactone treatment did not affect HRV variables, and although Lin et al. had a larger sample size than our study, differences in dosing and treatment duration should be noted. In congestive heart failure, and consistent with our results, spironolactone $50-100 \mathrm{mg}$ daily increased SDNN, SDANN (31) as well as $\mathrm{HF}$ and LF/HF (32) after 4-8 weeks of treatment. Although results from ESRD patients are not consistent, an increase in HRV has been found in multiple studies in different populations, indicating a beneficial effect of spironolactone on cardiac autonomic function as measured by HRV.

Contrary to our expectations and previous findings by Flevari et al. (30), we did not observe blood pressure reduction with spironolactone, but rather a slight but not statistically significant increase. It should be noted that the participants in the study by Flevari et al. had more extensive use of antihypertensive agents at baseline, and a slightly longer intervention period (4 months), which may explain the differences.

The use of MRAs in individuals with reduced renal function is limited by the risk of hyperkalemia (33). In this study, we observed no cases of severe hyperkalemia, and the treatment did not affect mean plasma potassium concentration. Multiple studies of spironolactone in ESRD have been conducted, each one individually not showing an increased risk of severe hyperkalemia in spironolactone treatment, although a recent meta-analysis weighted estimate indicates that the risk is increased (34). In consequence, and despite the lack of effect on potassium in this study, the risk of hyperkalemia during spironolactone treatment in ESRD should be recognised and appropriate monitoring conducted.

Some limitations apply to this study. Although the number of patients analysed was sufficient based on the power analysis, our sample size was limited. However, the crossover design increases statistical power, and we emphasise that according to 
the power calculation performed before study start, the analysis has sufficient power for detecting relevant changes in the primary outcome of PVCs. For secondary outcomes, the sample size may have been too small to show relevant effects, although it should be noted that all HRV variables measured showed a trend towards increasing values after treatment, which is concordant with the finding of statistically significant increases in SDNN and SDANN. Further, we did not use placebo during the control period, which allows for the theoretical possibility of an anticipation effect among participants. However, it is unlikely that such an effect would affect ECG variables and we employed blinded assessment of ECG recordings to avoid assessment bias. An additional limitation is that only four participants were female, and results could therefore not be analysed separately by sex. We also acknowledge the multiplicity of analyses and emphasise that change in PVCs was postulated as the primary endpoint in this study and that all analyses were specified $a$ priori. Strengths of the study include treatment randomisation, blinded assessment of outcomes and the crossover design, which limits confounding and increases statistical power. Also, this study included HD patients with and without congestive heart failure and may, therefore, be valid for a relatively unselected HD population.

We conclude that improved cardiac autonomic function, as indicated by an increased HRV, may contribute to the survival benefit from spironolactone treatment seen in previous studies in HD patients $(2,3)$. However, an increase in PVCs was observed and could indicate a possibly harmful effect with an increased risk of arrhythmias. These findings mandate cautious dosing of spironolactone or LTECG monitoring in subgroups at especially high risk of arrhythmia or sudden cardiac death.

\section{Acknowledgements}

Johan Sundholm assisted with planning, project administration and investigation.

\section{Disclosure statement}

The authors report no conflict of interest.

\section{Funding}

This work was supported by the Uppsala-Örebro Regional Research Council under Grants RFR-309221, RFR-480961 and RFR-562621; Region Örebro County Research Committee under Grants OLL-213701, OLL-261511 and OLL-329591; The Swedish Kidney Foundation; and The CUWX Counties Renal Patients' Association.

\section{Notes on contributors}

Michael Eklund, MD, PhD student in medical sciences at the School of Medical Sciences, Örebro University, Örebro, Sweden.
Olof Hellberg, MD, PhD in medical sciences. Retired researcher at the School of Medical Sciences, Örebro University, Örebro, Sweden.

Hans Furuland, MD, PhD in medical sciences, Department of Medical Sciences, Uppsala University Hospital, Uppsala, Sweden.

Yang Cao, PhD in biostatistics. Associate professor in biostatistics and epidemiology at the Unit of Clinical Epidemiology and Biostatistics, School of Medical Sciences, Örebro University, Örebro, Sweden. Researcher at the Unit of Integrative Epidemiology, Institute of Environmental Medicine, Karolinska Institutet, Stockholm, Sweden.

Erik Nilsson, MD, PhD in medical sciences. Researcher at the School of Medical Sciences, Örebro University, Örebro, Sweden, and researcher at the Department of Medical Epidemiology and Biostatistics, Karolinska Institutet, Stockholm, Sweden.

\section{ORCID}

Michael Eklund (D) https://orcid.org/0000-0001-7706-1758

Olof Hellberg

Hans Furuland (D) http://orcid.org/0000-0002-9001-614X

Yang Cao (i) http://orcid.org/0000-0002-3552-9153

Erik Nilsson (D) http://orcid.org/0000-0001-6968-6934

\section{References}

1. Sarnak MJ, Levey AS, Schoolwerth AC, Coresh J, Culleton B, Hamm LL, et al. Kidney disease as a risk factor for development of cardiovascular disease: a statement from the American Heart Association Councils on Kidney in Cardiovascular Disease, High Blood Pressure Research, Clinical Cardiology, and Epidemiology and Prevention. Hypertension. 2003;42:1050-65. doi: 10.1161/01.HYP.0000102971.85504.7c

2. Lin C, Zhang $\mathrm{Q}$, Zhang H, Lin A. Long-term effects of low-dose spironolactone on chronic dialysis patients: a randomized placebo-controlled study. J Clin Hypertens (Greenwich). 2016;18:121-8. doi: 10.1111/ jch. 12628

3. Matsumoto $Y$, Mori Y, Kageyama S, Arihara K, Sugiyama T, Ohmura $\mathrm{H}$, et al. Spironolactone reduces cardiovascular and cerebrovascular morbidity and mortality in hemodialysis patients. J Am Coll Cardiol. 2014;63:528-36. doi: 10.1016/j.jacc.2013.09.056

4. Chung YW, Yang YH, Wu CK, Yu CC, Juang JM, Wang YC, et al. Spironolactone is associated with reduced risk of new-onset atrial fibrillation in patients receiving renal replacement therapy. Int J Cardiol. 2016;202:962-6. doi: 10.1016/j.ijcard.2015.05.167

5. Kimura S, Ito M, Tomita M, Hoyano M, Obata H, Ding L, et al. Role of mineralocorticoid receptor on atrial structural remodeling and inducibility of atrial fibrillation in hypertensive rats. Hypertens Res. 2011;34:584-91. doi: 10.1038/hr.2010.277

6. Ouvrard-Pascaud A, Sainte-Marie Y, Bénitah JP, Perrier R, Soukaseum C, Nguyen Dinh Cat A, et al. Conditional mineralocorticoid receptor expression in the heart leads to life-threatening arrhythmias. Circulation. 2005;111:3025-33. doi: 10.1161/circulationaha.104.503706

7. Task Force of the European Society of Cardiology and the North American Society of Pacing and Electrophysiology. Heart rate variability: standards of measurement, physiological interpretation and clinical use. Circulation. 1996;93:1043-65. doi: 10.1161/01.CIR.93.5.1043

8. Hillebrand S, Gast KB, de Mutsert R, Swenne CA, Jukema JW, Middeldorp $\mathrm{S}$, et al. Heart rate variability and first cardiovascular event in populations without known cardiovascular disease: meta-analysis and 
dose-response meta-regression. Europace. 2013;15:742-9. doi: 10.1093/ europace/eus341

9. Inohara T, Kohsaka S, Okamura T, Watanabe M, Nakamura Y, Higashiyama A, et al. Long-term outcome of healthy participants with atrial premature complex: a 15-year follow-up of the NIPPON DATA 90 cohort. PLoS One. 2013;8:e80853. doi: 10.1371/journal.pone.0080853

10. Lin CY, Chang SL, Lin YJ, Lo LW, Chung FP, Chen YY, et al. Long-term outcome of multiform premature ventricular complexes in structurally normal heart. Int J Cardiol. 2015;180:80-5. doi: 10.1016/j.ijcard.2014.11.110

11. Qureshi W, Shah AJ, Salahuddin T, Soliman EZ. Long-term mortality risk in individuals with atrial or ventricular premature complexes (results from the Third National Health and Nutrition Examination Survey). Am J Cardiol. 2014;114:59-64. doi: 10.1016/j.amjcard.2014.04.005

12. Chong BH, Pong V, Lam KF, Liu S, Zuo ML, Lau YF, et al. Frequent premature atrial complexes predict new occurrence of atrial fibrillation and adverse cardiovascular events. Europace. 2012;14:942-7. doi: 10.1093/ europace/eur389

13. Meyerfeldt U, Wessel N, Schütt H, Selbig D, Schumann A, Voss A, et al. Heart rate variability before the onset of ventricular tachycardia: differences between slow and fast arrhythmias. Int J Cardiol. 2002;84:14151. doi: 10.1016/s0167-5273(02)00139-0

14. Santoro F, Di Biase L, Hranitzky P, Sanchez JE, Santangeli P, Perini AP, et al. Ventricular fibrillation triggered by PVCs from papillary muscles: clinical features and ablation. J Cardiovasc Electrophysiol. 2014;25:1158-64. doi: $10.1111 /$ jce. 12478

15. Barr CS, Lang CC, Hanson J, Arnott M, Kennedy N, Struthers AD. Effects of adding spironolactone to an angiotensin-converting enzyme inhibitor in chronic congestive heart failure secondary to coronary artery disease. Am J Cardiol. 1995;76:1259-65. doi: 10.1016/s0002-9149(99)80353-1

16. Gao X, Peng L, Adhikari CM, Lin J, Zuo Z. Spironolactone reduced arrhythmia and maintained magnesium homeostasis in patients with congestive heart failure. J Card Fail. 2007;13:170-7. doi: 10.1016/j. cardfail.2006.11.015

17. Ramires FJ, Mansur A, Coelho O, Maranhão M, Gruppi CJ, Mady C, et al. Effect of spironolactone on ventricular arrhythmias in congestive heart failure secondary to idiopathic dilated or to ischemic cardiomyopathy. Am J Cardiol. 2000;85:1207-11. doi: 10.1016/s0002-9149(00)00729-3

18. Sica DA. Pharmacokinetics and pharmacodynamics of mineralocorticoid blocking agents and their effects on potassium homeostasis. Heart Fail Rev. 2005;10:23-9. doi: 10.1007/s10741-005-2345-1

19. Rodríguez-Liñares L, Méndez AJ, Lado MJ, Olivieri DN, Vila XA, GómezConde I. An open source tool for heart rate variability spectral analysis. Comput Methods Programs Biomed. 2011;103:39-50. doi: 10.1016/j. cmpb.2010.05.012

20. Senn S. Cross-over trials in clinical research. Chichester, UK: John Wiley \& Sons; 2002. doi: 10.1002/0470854596

21. Nordeen SK, Bona BJ, Beck CA, Edwards DP, Borror KC, DeFranco DB. The two faces of a steroid antagonist: when an antagonist isn't. Steroids. 1995;60:97-104. doi: 10.1016/0039-128x(94)00001-s

22. Massaad $C$, Lombès $M$, Aggerbeck $M$, Rafestin-Oblin ME, Barouki R. Cell-specific, promoter-dependent mineralocorticoid agonist activity of spironolactone. Mol Pharmacol. 1997;51:285-92. doi: 10.1124/ mol.51.2.285

23. Lincevicius GS, Shimoura CG, Nishi EE, Perry JC, Casarini DE, Gomes GN, et al. Aldosterone contributes to sympathoexcitation in renovascular hypertension. Am J Hypertens. 2015;28:1083-90. doi: 10.1093/ajh/ hpu300

24. Kienzle MG, Ferguson DW, Birkett CL, Myers GA, Berg WJ, Mariano DJ. Clinical, hemodynamic and sympathetic neural correlates of heart rate variability in congestive heart failure. Am J Cardiol. 1992;69:761-7. doi: 10.1016/0002-9149(92)90502-p

25. Kaye DM, Lefkovits J, Jennings GL, Bergin P, Broughton A, Esler MD. Adverse consequences of high sympathetic nervous activity in the failing human heart. J Am Coll Cardiol. 1995;26:1257-63. doi: 10.1016/0735-1097(95)00332-0

26. Musch MW, Lucioni A, Chang EB. Aldosterone regulation of intestinal $\mathrm{Na}$ absorption involves SGK-mediated changes in NHE3 and $\mathrm{Na}+$ pump activity. Am J Physiol Gastrointest Liver Physiol. 2008;295:G909-19. doi: 10.1152/ajpgi.90312.2008

27. Shah NC, Pringle SD, Donnan PT, Struthers AD. Spironolactone has antiarrhythmic activity in ischaemic cardiac patients without cardiac failure. J Hypertens. 2007;25:2345-51. doi: 10.1097/ HJH.0b013e3282e9a72d

28. Wei J, Ni J, Huang D, Chen M, Yan S, Peng Y. The effect of aldosterone antagonists for ventricular arrhythmia: a meta-analysis. Clin Cardiol. 2010;33:572-7. doi: 10.1002/clc.20762

29. Karaboyas A, Zee J, Brunelli SM, Usvyat LA, Weiner DE, Maddux FW, et al. Dialysate potassium, serum potassium, mortality, and arrhythmia events in hemodialysis: results from the Dialysis Outcomes and Practice Patterns Study (DOPPS). Am J Kidney Dis. 2017;69:266-77. doi: 10.1053/j.ajkd.2016.09.015

30. Flevari P, Kalogeropoulou S, Drakou A, Leftheriotis D, Panou F, Lekakis J, et al. Spironolactone improves endothelial and cardiac autonomic function in non heart failure hemodialysis patients. J Hypertens. 2013;31:1239-44. doi: 10.1097/HJH.0b013e32835f955c

31. MacFadyen RJ, Barr CS, Struthers AD. Aldosterone blockade reduces vascular collagen turnover, improves heart rate variability and reduces early morning rise in heart rate in heart failure patients. Cardiovasc Res. 1997;35:30-4. doi: 10.1016/s0008-6363(97)00091-6

32. Yee KM, Pringle SD, Struthers AD. Circadian variation in the effects of aldosterone blockade on heart rate variability and QT dispersion in congestive heart failure. J Am Coll Cardiol. 2001;37:1800-7. doi: 10.1016/ s0735-1097(01)01243-8

33. Vardeny O, Wu DH, Desai A, Rossignol P, Zannad F, Pitt B, et al. Influence of baseline and worsening renal function on efficacy of spironolactone in patients With severe heart failure: insights from RALES (Randomized Aldactone Evaluation Study). J Am Coll Cardiol. 2012;60:2082-9. doi: 10.1016/j.jacc.2012.07.048

34. Quach K, Lvtvyn L, Baigent C, Bueti J, Garg AX, Hawley C, et al. The safety and efficacy of mineralocorticoid receptor antagonists in patients who require dialysis: a systematic review and meta-analysis. Am J Kidney Dis. 2016;68:591-8. doi: 10.1053/j.ajkd.2016.04.011 